\title{
Atomabsorptionsmessungen von Blei, Cadmium, Kupfer, Eisen und Zink im Seston der Ostsee
}

\author{
H.-P. WEIGEL \\ Biologische Anstalt Helgoland (Meeresstation); \\ Helgoland, Bundesrepublik Deutscbland
}

\begin{abstract}
Atomic absorption measurements of lead, cadmium, copper, iron and zinc in seston of the Baltic Sea. During a cruise with R. V. "Alkor" from Kiel Bight to Finnish Bay, nearly 100 seston samples were taken during April/May 1973. Samples from the 23 stations were analyzed for lead, cadmium, copper, iron and zinc by flameless atomic absorption spectrometry. The mean values for lead and cadmium were 123 and $5.8 \mathrm{ppm}$, respectively; for zinc, $733 \mathrm{ppm}$, for copper, $61 \mathrm{ppm}$, and for iron, $3535 \mathrm{ppm}$. In contrast to trace-metal analyses of off shore seston, the concentrations for lead and cadmium in Baltic-Sea seston are very high; these results are in good agreement with analyses of the dissolved metals. The concentrations of zinc, copper and iron in the particulate matter from the Baltic-Sea, apart from some local influences, agree well with values published from the concentrations in off shore seston. The distribution and variation of the individual values are discussed. The method for analysing the metals and its precision are briefly described.
\end{abstract}

\section{EINLEITUNG}

Die elementare Zusammensetzung mariner Organismen beschäftigt schon lange Biologen und Chemiker (Noddack \& Noddack, 1939; Vinogradov, 1953). Die Frage nach den am Aufbau der Organismen beteiligten Elementen, ihren Konzentrationsverhältnissen im Organismus und nach ihren metabolischen Funktionen stehen dabei im Vordergrund der Untersuchungen. Die meisten dieser Arbeiten bezogen sich jedoch auf die Hauptbestandteile des Meerwassers.

Nach den ersten spektakulären Vergiftungserscheinungen, hervorgerufen durch den Genuß von Quecksilber und Cadmium verseuchter Nahrung aus dem Meer (КовауаSH, 1969), schenkte man dem Problem der Anreicherung von Spurenmetallen mehr Bedeutung. Denn sobald Schadstoffe vom Organismus aufgenommen werden, haben die aus physikalischen Gesetzen abgeleiteten Berechnungen über ihre Ausbreitung und Verdünnung keine Berechtigung mehr. So publizierten Martin \& KnaUer (1972), Windom (1972), Preston (1973) und andere Autoren umfangreiche Studien über den Gehalt von Spurenmetallen in der partikulären Substanz. Für den Ostseeraum muß jedoch festgestellt werden, daß entsprechend analytische Anstrengungen bisher kaum unternommen wurden. Daten über die Metallkonzentration im Wasser veröffentlichten bisher nur KorolefF (1968), Sen GUPTA (1972), Kremling (1973) sowie Brüg- 
MANN (1974), und über die Anreicherung der Metalle im Seston liegen bisher gar keine Ergebnisse vor.

Die versäumten Untersuchungen erscheinen um so schwerwiegender, da die Ostsee als fast abgeschlossenes Randmeer ähnlichen Belastungen ausgesetzt zu sein scheint wie die offene Nordsee. Zwar empfängt die Ostsee kleinere Mengen an Abfallstoffen, jedoch ist bei ihr die Wassererneuerung wesentlich geringer und damit die Gefahr einer Verschmutzung genau so groß.

Die vorliegende Arbeit ist ein erster Bericht über die Verteilung einiger Spurenmetalle im Seston. Sie enthält Ergebnisse der Blei-, Kupfer-, Cadmium-, Eisen- und Zinkkonzentrationen während einer Ostseefahrt mit dem F. K. "Alkor" im April/ Mai 1973.

\section{METHODEN}

\section{Probennahme}

Auf 23 Stationen (Abb. 1) wurden ca. 100 Proben mit einem 2-Liter-Nansenschöpfer genommen. Da Rostpartikel des Winschdrahtes sowie Teile der Außenbordfarbe des Schiffes eine Kontamination und damit eine Verfälschung der Analysenergebnisse hervorrufen können, wurde auf die Entnahme einer Oberflächenprobe verzichtet.

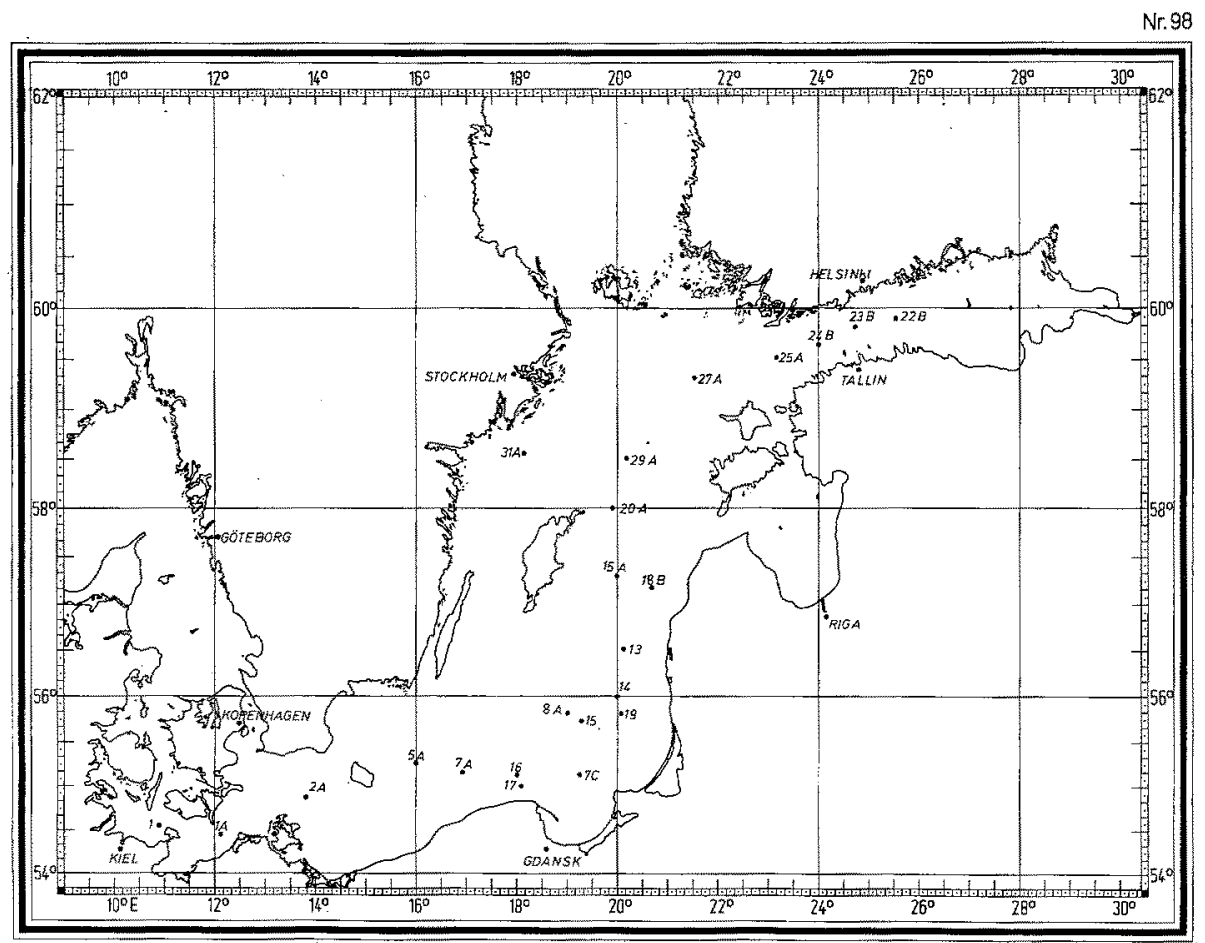

Abb. 1: Untersuchte Stationen in der Ostsee 
$500 \mathrm{ml}$ Probenwasser wurden durch ein $300-\mu$-Netz vorfiltriert, um in der Probe vorhandene größere Zooplankter auszusondern. Als Filtriergestell für den eigentlichen Filtrationsvorgang diente ein Schleicher- und Schüll-Glasfiltrationsgestell, das in MIBK/APCD gereinigt worden war.

Das Probenwasser wurde bei Unterdruck über ausgewogene Nuclepore-Filter (General Electric, USA) mit einem Durchmesser von $47 \mathrm{~mm}$ und einer Porenweite von $0,4 \mu$ filtriert. Die Filter wurden nach Beendigung des Filtrationsprozesses $2 \mathrm{mal}$ mit Austauscherwasser nachgespült (BRöcKEL, 1973), in gesäuberte Glasgefäße überführt und bis zur Analyse tiefgefroren aufbewahrt.

\section{Analytische Verfahren}

Die partikuläre Substanz wird heute ausschließlich durch einen naßchemischen Aufschluß zerstört (MARTIN \& KNAUER 1972; Windom, 1972; Nix \& Godwin, 1970). Die Nachteile dieser Methodik sind deutlich: (a) durch die Analyse mittels Flammenatomabsorption werden auf Grund der niederen Nachweisgrenzen größere Probenmengen exforderlich; (b) durch das Veraschen mit den notwendigen Chemikalien werden weitere Mengen des zu analysierenden Elementes in die Probe eingeschleppt;
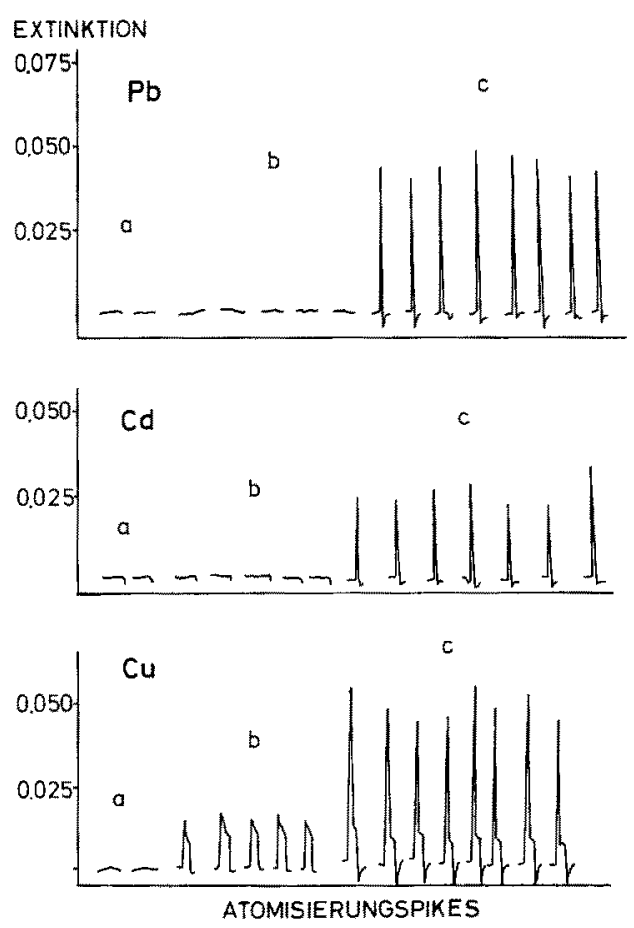

Abb. 2: Vergleich der Atomisierungspikes für die Schwermetalle in destilliertem Chloroform (a), in fünf verschiedenen Nuclepor-Filtern (b), und in einer Sestonprobe (c), die mehrmals nacheinander analysiert wurde 
(c) durch die Anwesenheit der Säuren werden die Atomisierungssignale bis zu 30\% unterdrückt; (d) es kann ein von GoRuscH (1959) festgestellter Verlust an Spurenmetallen auftreten.

Dieser Methodik liegt daher auch ein hoher Blindwert zugrunde; es ist notwendig, viel Probenwasser zu filtrieren, oder die Proben müssen mit Netzfängen genommen werden, wobei jedoch keine genauen Aussagen über die vertikale Verteilung der Metallkonzentration in der partikulären Substanz gemacht werden können.

Sowohl der große Zeitaufwand bei der Filtration des Probenwassers als auch der während des Veraschungsprozesses wirkt sich als weiterer Nachteil aus. Aus diesem Grunde wurde eine Methode entwickelt, die gewährleistet, daß die Probenvorbereitung nur wenig Zeit in Anspruch nimmt und die auch die Analyse kleinerer Probenmengen erlaubt.

Ein Perkin-Elmer-Modell-403-Atomabsorptionsspektrometer wurde zur Analyse benutzt. Da die Temperatur der Atomisierungszelle stufenlos regulierbar ist, wurde der Aufschluß der partikulären Substanz in der Zelle direkt durchgeführt.

Als Filter wurden Nuclepore-Filter verwendet, sowohl wegen ihres geringen Metallgehaltes (Abb. 2) und da sie frei von Auswascheffekten sind.

Ein weiterer Vorteil dieser Filter ist ihre gute Löslichkeit in Chloroform, das durch einmalige Destillation schnell von Metallen gereinigt werden kann (Abb. 2).

$500-1000 \mathrm{ml}$ Probenwasser wurden filtriert und Filter und Seston über Nacht bei $70^{\circ} \mathrm{C}$ getrocknet. Die Filter wurden in Quarzkolben überführt, $10 \mathrm{ml}$ Chloroform hinzugefügt, die Probe zum Homogenisieren $30 \mathrm{sec}$ einem Ultraschallbad ausgesetzt und die Suspension in die Küvette des Spektrometers injiziert und dort verascht. Zur Berechnung des Metallgehaltes in den Proben wurde der interne Standard benutzt. Die Präzision der gesamten Methode liegt für die hier analysierten Metalle zwischen 7 und $15 \%$. Ausführlich ist diese Methode von Weigel \& KREMLing (1975) beschrieben worden.

\section{ERGEBNISSE UND DISKUSSION}

Die Einzelergebnisse einiger Stationen sind in Tabelle 1 wiedergegeben. Auf Grund der relativ kleinräumigen Heterogenität konnten bei allen fünf Spurenmetallen keine großräumigen Gliederungen in der Konzentration im Seston unterschieden werden. Bemerkenswert ist diese Tatsache, da die dem Landeinfluß stärker ausgesetzten Areale keine wesentlich höhere Konzentration aufweisen als die zentrale Ostsee. Für die Metalle Blei und Cadmium ist ihre Verteilung auf dem Längsschnitt in Abbildung 3 wiedergegeben.

\section{B lei}

Die mittlere Konzentration des partikulär gebundenen Bleis beträgt $123 \mathrm{ppm}$ (Tab. 2). Demgegenüber stehen Werte von Martin \& Knauer (1972) aus der Montary Bay mit 1,22 ppm für Misch- bzw. 29,4 ppm für Mikroplankton. Windom (1972) analysierte Konzentrationen von $32 \mathrm{ppm}$ für das Phytoplankton vor der Südostküste 
Tabelle 1

Metallkonzentrationen im Seston der Ostsee (in ppm) auf sieben ausgewählten Stationen

\begin{tabular}{|c|c|c|c|c|c|c|}
\hline Station & Tiefe $(\mathrm{m})$ & $\mathrm{Pb}$ & $\mathrm{Cu}$ & $\mathrm{Cd}$ & $\mathrm{Fe}$ & $\mathrm{Zn}$ \\
\hline $\begin{array}{c}54^{\circ} 36^{\prime} \mathrm{N} \\
11^{\circ} 09^{\prime} \mathrm{E} \\
1\end{array}$ & $\begin{array}{r}5 \\
10 \\
20 \\
25\end{array}$ & $\begin{array}{r}302 \\
186 \\
71 \\
60\end{array}$ & $\begin{array}{l}80 \\
62 \\
23 \\
35\end{array}$ & $\begin{array}{l}1,8 \\
8,5 \\
3,0 \\
6,2\end{array}$ & $\begin{array}{l}9026 \\
5094 \\
2792 \\
4068\end{array}$ & $\begin{array}{l}948 \\
214 \\
217 \\
450\end{array}$ \\
\hline $\begin{array}{c}55^{\circ} 00^{\prime} \mathrm{N} \\
14^{\circ} 05^{\prime} \mathrm{E} \\
2 \mathrm{~A}\end{array}$ & $\begin{array}{r}5 \\
10 \\
20 \\
40\end{array}$ & $\begin{array}{r}80 \\
194 \\
359 \\
20\end{array}$ & $\begin{array}{r}151 \\
89 \\
97 \\
22\end{array}$ & $\begin{array}{l}7,3 \\
1,7 \\
4,9 \\
5,5\end{array}$ & $\begin{array}{l}7582 \\
3187 \\
6865 \\
3655\end{array}$ & $\begin{array}{r}844 \\
4758 \\
3150 \\
908\end{array}$ \\
\hline $\begin{array}{c}55^{\circ} 15^{\prime} \mathrm{N} \\
17^{\circ} 04^{\prime} \mathrm{E} \\
7 \mathrm{~A}\end{array}$ & $\begin{array}{r}5 \\
10 \\
20 \\
40 \\
50 \\
60 \\
90\end{array}$ & $\begin{array}{r}139 \\
157 \\
53 \\
35 \\
109 \\
115\end{array}$ & $\begin{array}{l}53 \\
37 \\
70 \\
25 \\
61 \\
37\end{array}$ & $\begin{array}{r}7,7 \\
3,4 \\
11,0 \\
2,9 \\
2,2 \\
2,1 \\
5,8\end{array}$ & $\begin{array}{r}1295 \\
3197 \\
5579 \\
4058 \\
- \\
1205 \\
2225\end{array}$ & $\begin{array}{r}165 \\
282 \\
344 \\
214 \\
- \\
194 \\
115\end{array}$ \\
\hline $\begin{array}{c}57^{\circ} 20^{\prime} \mathrm{N} \\
20^{\circ} 03^{\prime} \mathrm{E} \\
15 \mathrm{~A}\end{array}$ & $\begin{array}{r}5 \\
20 \\
50 \\
100 \\
125 \\
150 \\
175 \\
200 \\
225 \\
230\end{array}$ & $\begin{array}{l}34 \\
25 \\
46 \\
45 \\
65 \\
6,5 \\
6,1 \\
17 \\
14 \\
40\end{array}$ & $\begin{array}{r}12 \\
17 \\
10 \\
10 \\
16 \\
0 \\
9 \\
76 \\
2 \\
39\end{array}$ & $\begin{array}{l}1,7 \\
1,4 \\
0,7 \\
1,4 \\
1,4 \\
0,4 \\
0,3 \\
0,9 \\
0,5 \\
0,9\end{array}$ & $\begin{array}{r}429 \\
2970 \\
8086 \\
1938 \\
432 \\
1329 \\
748 \\
1055 \\
165 \\
1165\end{array}$ & $\begin{array}{r}183 \\
697 \\
447 \\
411 \\
177 \\
306 \\
115 \\
80 \\
47 \\
325\end{array}$ \\
\hline $\begin{array}{c}58^{\circ} 00^{\prime} \mathrm{N} \\
19^{\circ} 54^{\prime} \mathrm{E} \\
20 \mathrm{~A}\end{array}$ & $\begin{array}{r}5 \\
10 \\
20 \\
35 \\
50 \\
75 \\
100 \\
125 \\
150 \\
170\end{array}$ & $\begin{array}{r}128 \\
67 \\
106 \\
84 \\
35 \\
49 \\
53 \\
82 \\
0 \\
156\end{array}$ & $\begin{array}{r}27 \\
74 \\
224 \\
72 \\
72 \\
172 \\
25 \\
156 \\
142 \\
136\end{array}$ & $\begin{array}{r}6,8 \\
12,8 \\
28,1 \\
4,7 \\
4,3 \\
4,1 \\
5,2 \\
15,3 \\
4,0 \\
8,0\end{array}$ & $\begin{array}{r}1709 \\
1116 \\
3766 \\
1396 \\
2998 \\
4134 \\
405 \\
5470 \\
5479 \\
557\end{array}$ & $\begin{array}{r}1928 \\
1800 \\
20845 \\
787 \\
1060 \\
823 \\
843 \\
1812 \\
1059 \\
986\end{array}$ \\
\hline $\begin{array}{c}58^{\circ} 53^{\prime} \mathrm{N} \\
20^{\circ} 19^{\prime} \mathrm{E} \\
29 \mathrm{~A}\end{array}$ & $\begin{array}{r}5 \\
10 \\
25 \\
50 \\
100 \\
125 \\
150 \\
160\end{array}$ & $\begin{array}{r}334 \\
313 \\
351 \\
149 \\
44 \\
216 \\
395 \\
0,0\end{array}$ & $\begin{array}{c}52 \\
354 \\
51 \\
38 \\
14 \\
19 \\
0,0 \\
17\end{array}$ & $\begin{array}{r}10,4 \\
7,0 \\
7,3 \\
4,5 \\
2,8 \\
7,7 \\
17,6 \\
5,6\end{array}$ & $\begin{array}{r}4630 \\
11272 \\
2392 \\
7086 \\
2176 \\
6166 \\
10481 \\
2673\end{array}$ & $\begin{array}{r}478 \\
80 \\
272 \\
166 \\
81 \\
80 \\
295 \\
188\end{array}$ \\
\hline $\begin{array}{c}58^{\circ} 35^{\prime} \mathrm{N} \\
18^{\circ} 14^{\prime} \mathrm{E} \\
31 \mathrm{~A}\end{array}$ & $\begin{array}{r}5 \\
10 \\
25 \\
50 \\
75 \\
100 \\
125 \\
150 \\
200 \\
295 \\
395 \\
425\end{array}$ & $\begin{array}{r}152 \\
219 \\
488 \\
132 \\
527 \\
960 \\
31.5 \\
200 \\
100 \\
96 \\
405 \\
298\end{array}$ & $\begin{array}{r}29 \\
32 \\
55 \\
10 \\
10 \\
408 \\
64 \\
140 \\
28 \\
69 \\
23 \\
81\end{array}$ & $\begin{array}{r}13,5 \\
1,4 \\
18,2 \\
7,9 \\
0,0 \\
8,4 \\
17,5 \\
14,0 \\
5,4 \\
6,7 \\
4,4 \\
16,8\end{array}$ & $\begin{array}{r}1310 \\
1917 \\
3058 \\
531 \\
5990 \\
1210 \\
- \\
1399 \\
905 \\
\overline{-}\end{array}$ & $\begin{array}{r}2365 \\
1536 \\
5941 \\
80 \\
471 \\
2250 \\
3550 \\
2432 \\
184 \\
665 \\
1104 \\
1557\end{array}$ \\
\hline
\end{tabular}



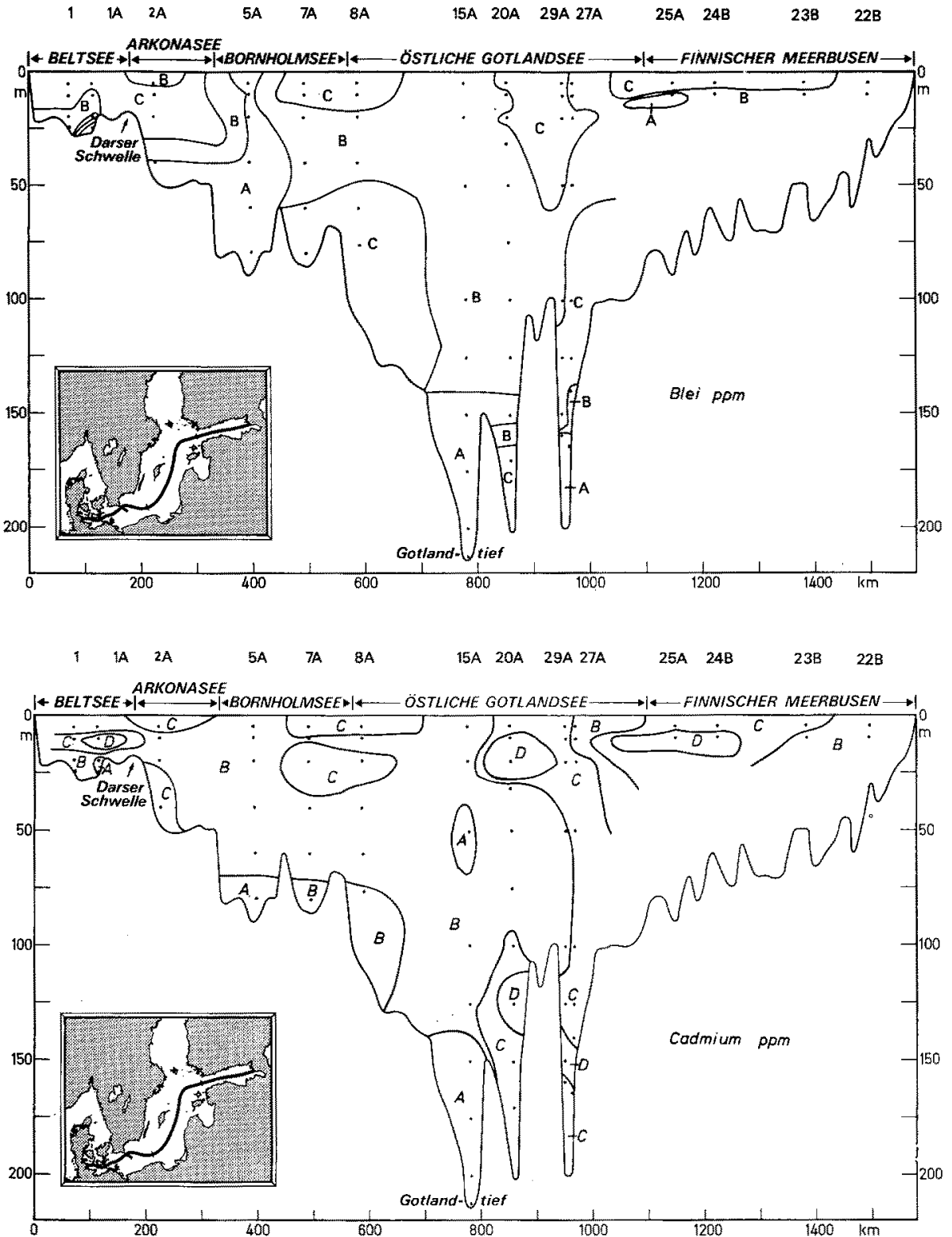

Abb. 3: Längsschnitte in der $\mathrm{Pb}$ - und $\mathrm{Cd}-K o n z e n t r a t i o n$ in der partikulären Substanz der Ostsee. (Konzentrationsbereiche $\mathrm{Pb}: \mathrm{A}=0-30 \mathrm{ppm}, \mathrm{B}=30-100 \mathrm{ppm}, \mathrm{C}=>100 \mathrm{ppm}$; $\mathrm{Cd}$ : $\mathrm{A}=0-1 \mathrm{ppm}, \mathrm{B}=1-5 \mathrm{ppm}, \mathrm{C}=5-10 \mathrm{ppm}, \mathrm{D}=>10 \mathrm{ppm})$

der USA und in der gleichen Größenordnung liegen die von Forster et al. (1972) publizierten Daten mit 44,4 ppm. Die Proben stammen aus der Karibischen See, setzen sich jedoch hauptsächlich aus Zooplankton zusammen. 
Tabelle 2

Mittlere Spurenmetallkonzentration im Seston der Ostsee

\begin{tabular}{|ccccc|}
\hline Metall & Probenzahl & $\begin{array}{c}\text { Mittelwert } \\
(\mathrm{ppm})\end{array}$ & $\begin{array}{c}\text { Relative Stan- } \\
\text { dardabweichung } \\
(\%)\end{array}$ & Bereich (ppm) \\
\hline $\mathrm{Pb}$ & 99 & 123 & \pm 102 & $0-527$ \\
$\mathrm{Cu}$ & 100 & 61 & \pm 110 & $0-354$ \\
$\mathrm{Cd}$ & 99 & 5,8 & \pm 92 & $0-28,1$ \\
$\mathrm{Zn}$ & 93 & 733 & \pm 139 & $28-4758$ \\
$\mathrm{Fe}$ & vgl. Tab. 3 & & & \\
\hline
\end{tabular}

Auch ohne Berïcksichtigung der extrem niederen Konzentration von 1,22 ppm, die MARTIN \& KNAUER veröffentlichten, weist die Bleikonzentration der partikulären Substanz der Ostsee stark überhöhte Werte auf. Diese Differenz läßt sich durch die verschiedenen Meßmethoden der zitierten Arbeiten nicht erklären. Ebenso gibt der Streuungsbereich der Einzelergebnisse keinen Aufschluß über die Ursache der Abweichung.

Unter Berücksichtigung der Bleikonzentration des Ostseewassers ergibt sich jedoch ein einheitliches Bild. KREMLING (1973) analysierte die Konzentration des gelösten Bleis mit $1,1 \mu \mathrm{g} / \mathrm{l}$, BrügManN (1974) mit $0,65 \mu \mathrm{g} / \mathrm{l}$, und Sen Gupta (1972) gibt einen Bereich $z$ wischen $O$ und $5,6 \mu \mathrm{g} / \mathrm{kg}$ an. Diese Ergebnisse weisen auf stark uberhöhte Konzentrationen im Ostseewasser hin, da Wasseranalysen des freien Ozeans wesentlich geringere Werte ergaben (RILEY \& Chester, 1971; TATsumoto \& PATTERSON, 1963; SChaule, 1974).

Eine Metallanreicherung ist unter anderem von den Konzentrationsverhältnissen im Wasser abhängig (GARDINER, 1974). Daher ist die hohe Bleikonzentration im Seston Folge der hohen Bleikonzentration des Ostseewassers.

Diese Aussage über eine Bleianreicherung in der Ostsee werden durch Untersuchungen von ERLENKÄUSER et al. (1974) unterstützt, die in den oberen $20 \mathrm{~cm}$ des Sedimentes der Kieler Bucht zur Oberfläche hin ansteigende Bleikonzentrationen nachweisen konnten.

\section{Cadmium}

Überhöhte Werte lassen auch die Analysen der Cadmiumkonzentration in der partikulären Substanz erkennen. Die mittlere Konzentration von 5,8 ppm (Tab. 2) ist im Vergleich zu den von Martin \& KNAUER (1972) und Windom (1972) publizierten Daten höher. Der um den Faktor 2 erhöhte Wert für die Ostsee spiegelt sich auch in den Konzentrationen des gelösten Cadmiums wider, denn BrügmanN (1974) und KREMLING (1973) geben fast übereinstimmend eine Konzentration von $0,2 \mu \mathrm{g} / 1$ an, während Riley und Chester (1971) sowie Chester \& Stoner (1974) geringere Konzentrationen für den Ozean publizierten. Für eine Kontamination der Ostsee durch Cadmium sprechen weiterhin die Ergebnisse von ERLENKÄUSER et al. (1974), die paral- 
lel zu Blei in den Sedimenten der Kieler Bucht ebenfalls erhöhte Cadmiumkonzentrationen ermittelten.

Die Vermutung, die erhöhten Konzentrationen durch eine Kontamination der Proben bei der Probennahme, Aufbewahrung sowie Vorbereitung zur Analyse erklären zu wollen, scheidet aus. Die überhöhten Konzentrationen im Wasser lassen eindeutig die Ursachen überhöhter Metallkonzentrationen im Seston erkennen.

\section{$\mathrm{Zink}$}

Die scheinbar erhöhten Zinkkonzentrationen in der partikulären Substanz (Tab. 2) können mit erhöhten Zinkkonzentrationen des Ostseewassers nicht erklärt werden. Die von Sen Gupta (1972) und Kremling (1973) publizierten Werte stehen in guter Übereinstimmung mit denen von Riley \& Chester (1971), Zirino \& Healy (1970), RILEY \& TAYler (1972).

Der hier analysierte Mittelwert von $733 \mathrm{ppm}$ liegt, verglichen mit Daten von 10,9 ppm (Martin \& KNAUER, 1972), 207 ppm (Windom, 1972 und Robertson et al., 1972) höher. Werden jedoch hier die Mittelwerte der einzelnen Stationen differenziert betrachtet, wird ersichtlich, wie stark diese untereinander variieren. Der Mittelwert für die Stationen $2 \mathrm{~A}, 7 \mathrm{C}, 20 \mathrm{~A}$ und $31 \mathrm{~A}$ beträgt $1576 \mathrm{ppm}$, der der andern hingegen nur $299 \mathrm{ppm}$. Dieser steht jedoch in guter Übereinstimmung mit den zitierten Arbeiten. MarTin \& KnaUer (1972) geben jedoch auch für Mikroplankton eine Konzentration von $1510 \mathrm{ppm}$ an, die mit der der vier genannten Stationen identisch ist. Diese Ergebnisse deuten auf starke lokale Einflüsse hin. Die Zinkkonzentration der partikulären Substanz kann aber dennoch als natürliche für die Ostsee angesehen werden.

\section{Kupfer}

Die Konzentration des partikulär gebundenen Kupfers beträgt 61 ppm (Tab. 2). Unter Berücksichtigung der Streuung, die auch andere Autoren publizierten (MARTrN \& KNAUER, 1972; WINDOM, 1972; Forster et al., 1972), müssen diese Konzentrationen als natürliche der partikulären Substanz der Ostsee angesehen werden. Die Analysen des gelösten Kupfers bestätigen diese Aussage (Rnley \& Chester, 1971; RILEY \& TAYLer, 1972; Sen Gupta, 1972; KremLing, 1973).

\section{Eisen}

Im Gegensatz zu den bisher diskutierten Metallen konnte ein signifikanter Unterschied in der Konzentration des partikulär gebundenen Eisens in der salzarmen Deckschicht der Ostsee und im Tiefenwasser ermittelt werden. Die mittlere Konzentration in der Deckschicht beträgt 4471 ppm, die des Tiefenwassers 2951 ppm. Erheblichen Einfluß auf die hohen Konzentrationen der Deckschicht haben die Stationen im Finnischen Meerbusen, die für sich eine Konzentration von 10000 ppm aufweisen (Tab. 3). 
Tabelle 3

Mittlere Fe-Konzentration in verschiedenen Arealen der Ostsee $(s=$ relative Standardabweichung, $N=$ Probenzahl)

\begin{tabular}{|cccl|}
\hline ppm & $\mathrm{s}(\%)$ & $\mathrm{N}$ & \multicolumn{1}{c|}{ Areale } \\
\hline 3616 & \pm 151 & 52 & Deckschicht ohne Finnischen Meerbusen \\
4471 & \pm 123 & 60 & Deckschicht mit Finnischen Meerbusen \\
10026 & \pm 53 & 8 & Finnischer Meerbusen \\
2951 & \pm 118 & 36 & Tiefenwasser \\
\hline
\end{tabular}

Derartige Unterschiede konnten auch von Gustavsson (persönliche Mitteilung) nachgewiesen werden und scheinen durch die Süßwasserzufuhw der Newa bedingt. Ohne Berücksichtigung dieser Konzentration reduziert sich diese in der Deckschicht auf 3616 ppm (Tab. 3). Ein Vergleich der Mittelwerte in der Deckschicht ohne den Finnischen Meerbusen und im Tiefenwasser ergibt einen signifikanten Unterschied. Aber auf Grund der Genauigkeit der Analyse, die für Eisen $12 \%$ beträgt, dürfen die beiden Konzentrationen als nicht voneinander unterschieden angesehen werden. Die mittlere Konzentration des partikulär gebundenen Eisens beträgt daher 3535 ppm. Ein Vergleich mit bisher publizierten Daten (MARTIN \& KNAUER, 1972; RoBERTSON et al., 1972; Forster et al., 1972) läßt die Daten dennoch überhöht erscheinen. Die Vermutung, diesen Effekt als Verunreinigung der Ostsee zu interpretieren, kann auf Grund der guten Übereinstimmung der Wasseranalysen der Ostsee und des Ozeans (KorolefF, 1968; MeNzel \& SPAEt H, 1962; Riley \& Chester, 1971) nicht aufrecht erhalten werden. Zum einen liegt Eisen neben dem in der partikulären Substanz angereicherten kolloidal als Eisenhydroxid bzw. Eisenphosphat yor (KoronefF, 1968). Diese Kolloide werden gleichzeitig mit der partikulären Substanz auf den Filtern angereichert und beeinflussen somit die Analyse der Eisenkonzentration der partikulären Substanz. Der hohe Süßwasserzufluß der Ostsee verstärkt diesen Einfluß. Hierin liegt auch die Ursache der erhöhten Eisenkonzentrationen im Seston, so daß die Ostseedaten keine Differenzen zu den zitierten Arbeiten aufweisen.

\section{ZUSAMMENFASSUNG}

1. Auf einer Forschungsfahrt in die Ostsee im April/Mai 1973 wurden 100 Sestonproben entnommen und auf ihren Gehalt an Blei, Cadmium, Zink, Kupfer und Eisen mit Hilfe der flammenlosen Atom-Absorptions-Spektrometrie analysiert.

2. Die mittlere Konzentration im Seston der Ostsee beträgt für Blei $123 \mathrm{ppm}$, für Cadmium 5,8 ppm, für Zink $300 \mathrm{ppm}$, für Kupfer $61 \mathrm{ppm}$ und für Eisen 3535 ppm.

3. Im Vergleich zu Metallanalysen ozeanischen Sestons müssen die Blei- und Cadmiumkonzentrationen als stark überhöht angesehen werden.

4. Die Konzentrationen des partikulär gebundenen Eisens, Zinks und Kupfers können bis auf lokal stark erhöhte Eisen- und Zinkkonzentrationen als natürlich angesehen werden.

5. Arbeitsweise und Meßgenauigkeit der Metallanalytik werden beschrieben. 
Danksagung. Der Autor dankt Herrn Dr. K. KREMLing für die Bereitstellung eines Atomabsorptionsspektrometers für die Zeit der Analysen.

\section{ZITIERTE LITERATUR}

BröcKEL, K. von, 1973. Eine Methode zur Bestimmung des Kaloriengehaltes von Seston. Kieler Meeresforsch. 29, 34-49.

BRÜGMANN, L., 1974. Die Bestimmung von Spurenelementen im Meerwasser unter Verwendung einer stationären Quecksilberelektrode. Acta hydrochim. hydrobiol. 2, 123-138.

Chester, R. \& STONER, J. H., 1974. The distribution of zinc, nickel, manganese, cadmium, cobalt and iron in some surface waters from the world. Mar. Chem. 2, 17-32.

Erlenkäuser, H., Suess, E. \& Wrllkomm, H., 1974. Industralization affects heavy metal and carbon isotope concentrations in recent Baltic Sea sediments. Geochim. Cosmochim. Acta 38, 823-842.

Forster, W. O., Wood, E. D. \& Padovant, F., 1972. A study program to identity problems related to oceanic environmental quality in the Carribbean. Baseline studies of pollutants in the marine environments. Workshop at Brookhaven, Nat. Lab., 275-324.

GARDINER, J., 1974. The chemistry of cadmium in natural waters-II. The adsorption of cadmium on river muds and naturally occuring splids. Wat. Res. 8, 157-164.

Gorusch, T. T., 1959. Radiodemical investigations on the recovery for analysis of trace elements in organic and biological materials. Analyt. Chem. 84, 135-173.

Kobayashr, L., 1969. Relation between Itai-Itai disease and the pollution of river water by cadmium from a mine. Advances in Water Pollution Research. Pergamon Pr., Oxford (1) I $25,1-7$.

KoRolefF, F., 1968. A note on the iron content of Baltic waters. Annual meeting of the International Council for Exploration of the Sea (= C.M. - I.C.E.S.) 34.

KremLing, K., 1973. Voltametrische Messungen über die Verteilung von Zink, Cadmium, Blei und Kupfer in der Ostsee. Kieler Meeresforsch. 29, 77-84.

MarTin, J. H. \& Knauer, G. A., 1972. A comparison of inshore vs. offshore levels of 21 trace and major elements in marine plankton. Baseline studies of pollutants in the marine environments, Workshop at Brookhaven, Nat. Lab., 35-66.

Menzel, D. H. \& Spaeth, J. P., 1962. Occurrence of iron in the Sargasso Sea off Bermuda. Limnol. Oceanogr. 7, 155-158.

Nx, J. \& GooDwrN, T., 1970. The simultaneous extraction of iron, manganese, copper, cobalt, nickel, chromium, lead and zinc from natural water for determination by Atomic Absorption Spectroscopy. Art. Absorp. Newsl. 9, 119-122.

Noddack, I. \& NoDdAck, W., 1939. Die Häufigkeiten der Schwermetalle in Meerestieren. Ark. Zool. 32A, 1-35.

PRESTON, A., 1973. Cadmium in the marine environment of the United Kingdom. Mar. Poll. Bull. 4, 105-107.

Riley, J. P. \& Chester, R., 1971. Introduction to marine chemistry. Acad. Pr., London, $465 \mathrm{pp}$.

- \& TAYLER, D., 1972. The concentrations of cadmium, copper, iron, manganese, molybdenum, nickel, vanadium and zine in part of the tropical north-east Atlantic Ocean. Deep-Sea Res. $19,307-317$.

ROBERTSON, D. E., 1968. Role of contamination in trace elements analysis of sea water. Analyt. Chem. 40, 1067-1072.

- Rancitelli, I. A., Langford, J. C. \& Perkins, R. W., 1972. Batelle-Northwest contribution to the IDEO Baseline Studies of pollutants in the marine environments, Workshop at Brookhaven, Nat. Lab., 231-274.

Schaule, B., 1974. Massenspektrometrische Konzentrationsbestimmungen von Blei in Meerwasser. Diplomarbeit, Universität Heidelberg, 68 pp.

SEN GUPTA, R., 1972. On some trace metals in the Baltic. Ambio 1, 226-230. 
Tatsumoto, M. T. \& Patterson, C. C., 1963. Concentration of common lead in some Atlantic and Mediterranean water and in snow. Nature, Lond. 199, 350-352.

Vinogradov, A. P., 1953. The elementary chemical composition of marine organisms. Mem. Sears Fdn. mar. Res. 2, 1-647.

WeIGEL, H. P. \& KREMLING, K., 1975. A new method for the determination of trace metals in seston by flameless atomic absorption spectrometry. Annual meeting of the International Council for the Exploration of the Sea (= C.M. - I.C.E.S.) E 39.

WINDom, H. L., 1972. Arsenic, cadmium, copper, lead, mercury and zinc in marine biota-North Atlantic Ocean. Baseline studies of pollutants in the marine environments. Workshop at Brookhaven, Nat. Lab. 121-148.

ZrRINo, A. \& HEALY, M. L., 1970. Voltametric measurements of zinc in the north-eastern tropical Pacific Ocean. Limnol. Oceanogr. 16, 773-778.

Anschrift des Autors: Dr. H.-P. WeigeL

Biologische Anstalt Helgoland (Meeresstation)

D-2192 Helgoland

Bundesrepublik Deutschland 